\title{
Hipótesis: Un paso central en el pensamiento clínico y la generación de evidencia científica
}

\author{
M. Rosario Acuña Quicones', Juvenal A. Ríos Leal', Tomás Labbé Atenas, ${ }^{1 *}$
}

1. Escuela de Medicina, Facultad de Medicina y Ciencia, Universidad San Sebastián. Santiago, Chile.

Hypothesis: an important step in the clinical thinking and the development of scientific evidence

Resumen: El entrenamiento en metodologías de la investigación refuerza diversas habilidades críticas para los profesionales de la salud. El planteamiento de hipótesis, lejos de ser un proceso trivial, constituye un paso fundamental para adquirir un adecuado razonamiento clínico. En el presente artículo, los conceptos de conjetura, hipótesis, teorías y pregunta de investigación son revisados y se enfatiza la importancia del criterio científico en la formación de las escuelas de salud.

Palabras clave: Bioestadística, Diseño de Investigación, Educación Médica.

Abstract: The training in research methods enhance several abilities that are critical for the health professionals. The adequate proposition of hypothesis, far from being a trivial process, constitutes a fundamental step for acquiring an adequate clinical reasoning. In this article, the concepts of conjecture, hypothesis theories and research question are reviewed and the relevance of the scientific criteria for health professionals is emphasized.

Keywords: Biostatistics, Medical Education, Research design.

Acuña MR., et al. Hipótesis: Un paso central en el pensamiento clínico y la generación de evidencia científicaRev Chil Radiol 2019; 25(3): 83-86.

${ }^{*}$ Correo electrónico: Tomás Labbé Atenas MD, PhD / tplabbe@uc.cl

Trabajo enviado el 30 de julio de 2019. Aceptado para publicación el 21 de agosto de 2019.

\section{Introducción}

Una importante transición hecha por los estudiantes de las carreras del área de la salud es el paso de la etapa pre-clínica a la etapa clínica ${ }^{(1)}$. Según Flexner, quien ya en su reporte de 1910 sugiere este cambio de escenario, el razonamiento analítico debe ser central en el entrenamiento intelectual de los médicos. El aprendizaje en clínica estimula a clínicos y estudiantes a investigar sobre las preguntas que surgen al cuidado de los pacientes. En este contexto, el aprendizaje de las metodologías de la investigación constituye una herramienta en la generación de un mejor cuidado del paciente ${ }^{(2)}$.

El razonamiento clínico experto puede entenderse como una consecuencia del conocimiento extenso y multidimensional(3). No obstante, el volumen de conocimiento no es suficiente para este proceso, sino que requiere de procesos fundamentales como la formulación de hipótesis. Estas hipótesis demandan de una extensa búsqueda y el análisis de información, tanto para ser comprobadas como refutadas. Este proceso se desarrolla con el fin de crear conocimiento nuevo útil para la práctica médica. Pese a lo anterior, con el conocimiento experto se puede llegar a un buen razonamiento clínico, pero el conocimiento teórico por sí solo no basta porque es imprescindible desarrollar pensamiento crítico, y el primer para ello es entrenar a los estudiantes en la adecuada formulación de las 
hipótesis que serán evaluadas.

El esqueleto conductor de todo proceso de investigación es la correcta formulación de hipótesis y la formulación de una metodología lógicamente relacionada. De esta manera, el objetivo del presente artículo es proveer una aproximación inicial a los conceptos de pregunta de investigación, hipótesis científica, hipótesis estadística o diagnóstica y teoría. Finalmente se explicará por qué una hipótesis tiene que ser funcional, en el sentido que tiene que dirigir la investigación de principio a fin, con el objetivo de crear un conocimiento nuevo útil.

\section{De Conjeturas a Teorías}

El conocimiento se maneja de forma diaria en diferentes niveles de complejidad, desde simples conjeturas hasta la representación sistemática de sistemas teóricos derivados de importantes procesos de investigación. Una conjetura corresponde a una idea que se genera a partir de un conocimiento incompleto sobre un tema. Importantes epistemólogos han apuntado a la tendencia de los científicos a operar en base a conjeturas, saltando a conclusiones incluso después de una simple observación, como una amenaza frecuente ${ }^{(4)}$. Por otra parte, una hipótesis corresponde a una aseveración susceptible de ser sometida a prueba mediante un cierto método experimental(5).

Por su parte, el concepto de teoría resulta ciertamente más esquivo. Habitualmente se acepta que, habiendo evolucionado de conjeturas a hipótesis, una vez que una suma importante de estas últimas en un determinado campo se sistematiza de manera coherente, se constituye una teoría. Esto proveerá un sustrato teórico que guíe y oriente las intervenciones e interpretaciones ${ }^{(6,7)}$.

\section{Preguntas de Investigación}

Antes de la formulación de una hipótesis resulta fundamental identificar la pregunta de investigación que se busca responder. Esta tiene como objetivo darle un propósito a un estudio y, asimismo, ayuda a delimitar la literatura sobre el tema a investigar. Para el profesional sanitario resulta crítico revisar literatura sobre el tema en cuestión, puesto que es la única forma de ubicar a cada interrogante en el contexto de la evidencia disponible ${ }^{(8)}$. Con fines pedagógicos, se ha propuesto el acrónimo PICO para recordar que una pregunta de investigación bien planteada debe explicitar la Población de interés, la Intervención a evaluar, la Comparación a realizar y el Outcome estudiado (salida o resultado evaluado)(9). Además, en la enseñanza de métodos de investigación suele incluirse el acrónimo FINER, enfatizando ciertas características globales que deben distinguir a las preguntas de investigación: Factible, Interesante, Nueva, Ética y Relevante. La figura 1 muestra la sistematización de las preguntas de investigación a través del acrónimo PICO y su evaluación por medio de la sigla FINER.

\section{Las diferentes formas de una hipótesis}

Desde la perspectiva psicológica, diagnosticar es un proceso de testear distintas hipótesis ${ }^{(10)}$. Muchas hipótesis diagnósticas se hacen incluso antes del primer contacto del médico con el paciente ${ }^{(11)}$. Este proceso de reconocer ciertos patrones que guían a una hipótesis diagnóstica depende de mecanismos neurofisiológicos estandarizados que tienden a automatizarse con la experiencia ${ }^{(12)}$. No obstante, el interés que reviste este tema, el complejo proceso de generación de hipótesis diagnóstica, aunque relacionado, excede el foco de este artículo.

Considerando que una hipótesis, o hipótesis científica, es aquella que se puede poner a prueba a través del método científico, llamamos hipótesis estadística a aquella que es contrastable mediante un constructo matemático en el proceso de "prueba de hipótesis"(13). Para desarrollar este contraste, se debe transformar un planteamiento científico en una expresión verbalmente acotada que, cumpliendo con ciertas características permitirá discriminar entre una aseveración nula y una alternativa. En esta línea, la hipótesis nula $(\mathrm{H} 0)$ es aquella que establece la ausencia de diferencias, asociación, correlación, interacción o el concepto que corresponda. En otras palabras, la hipótesis nula corresponde a los valores que conocemos y que esperamos obtener. Por otro lado, la hipótesis alternativa o del investigador $(\mathrm{H} 1)$ es aquella en la que se espera encontrar una diferencia respecto a lo conocido ${ }^{(14)}$. Frente a estas dos opciones se toma una decisión estadística.

Ya que en cualquier sistema hay variabilidad, es importante determinar si aquella variabilidad se debe al azar, o bien, si se debe a otro fenómeno ${ }^{(15)}$. El valor $P$ es la probabilidad de obtener, por el azar, un efecto igual o más extremo que el observado en la hipótesis nula que se presumió como verdadera ${ }^{(16)}$. Este valor funciona como punto de corte entre la dicotomía de $\mathrm{H} 1$ y H0, y es elegido por el investigador a priori. De esta manera, la decisión estadística será un proceso estandarizado. No obstante, conceptualmente se acepta que nos referimos a probabilidades y en ningún caso a certezas absolutas.

Adicionalmente, el proceso de formulación de hipótesis requiere que la información sea organizada y las propuestas hipotéticas estructuradas de tal manera de que el conjunto de $\mathrm{HO}$ y $\mathrm{H} 1$ cumplan con ciertas características fundamentales ${ }^{(17)}$ :

Exhaustividad: Deben cubrir todos los resultados posibles. El proceso de prueba de hipótesis no da lugar a un posible resultado que no sea clasificable como $\mathrm{H} 0$ o $\mathrm{H} 1$.

Excluyentes: Los conjuntos que dan cuenta del 
espectro de las Hipótesis H0 y H1 no comparten resultados que sean ambiguos entre ambas proposiciones.

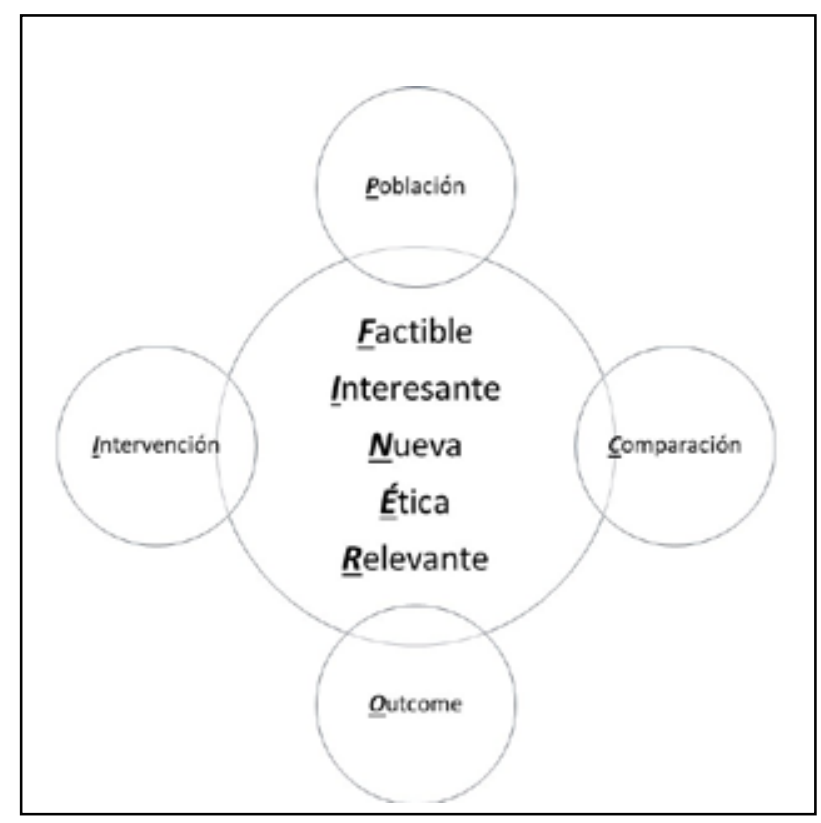

Figura 1. Componentes y criterios cualitativos de una pregunta de investigación. La figura muestra cómo cada componente metodológicamente identificable de una pregunta de investigación debe cumplir con los criterios de factibilidad, interés, novedad, ética y relevancia.

\section{Conclusiones}

Los diferentes procesos para la generación de preguntas de investigación e hipótesis científicas revisten una importancia que excede el impacto que la obtención de estas habilidades podría tener en la productividad científica individual. Por ello, el pensamiento científico y el aprendizaje de las metodologías de la investigación benefician a todos los profesionales sanitarios en formación.

La investigación es el campo de entrenamiento de muchas habilidades que deben desarrollar los médicos en su formación, entre ellas se encuentran el razonamiento clínico y el pensamiento crítico. Este vacío en el conocimiento debe ser remendado, pues un buen profesional investiga (en diferentes contextos y complejidades), y a partir de eso mejora el nivel de la atención que reciben sus pacientes ${ }^{(18)}$.

Transitar desde el manejo de conjeturas al abordaje de hipótesis constituirá una herramienta para el desarrollo del pensamiento crítico, puesto que se transita de una aproximación contemplativa a una analítica, reduciendo los potenciales errores asociados a un abordaje conjetural(19). Adicionalmente, habilidades como la tolerancia a la incertidumbre requerida para la resolución de un problema clínico, un acercamiento reflexivo a la información y creatividad para observar los problemas desde ángulos innovadores son beneficios de una educación médica que considera la investigación como un proceso central ${ }^{(7,20)}$.

\section{Referencias}

1. Teunissen PW. On the transfer of theory to the practice of research and education [(Internet). Medical Education. 2010; 44: 534-535. Available from: http://dx.doi. org/10.1111/j.1365-2923.2010.03637.x

2. Cooke M, Irby DM, Sullivan W, Ludmerer KM. American medical education 100 years after the Flexner report. N Engl J Med. 2006 sep 28; 355(13): 1339-1344.

3. Norman G. Research in clinical reasoning: past history and current trends. Med Educ. 2005 apr; 39(4): 418-427.

4. Popper KR, Hudson GE. Conjectures and Refutations [Internet]. Physics Today. 1963; 16: 80-82. Available from: http://dx.doi.org/10.1063/1.3050617

5. Deamer D. Conjecture and hypothesis: The importance of reality checks (Internet). Beilstein Journal of Organic Chemistry. 2017; 13: 620-624. Available from: http:// dx.doi.org/10.3762/bjoc.13.60

6. Schmidt HG, van der Arend A, Moust JH, Kokx I, Boon L. Influence of tutors' subject-matter expertise on student effort and achievement in problem-based learning. Acad Med. 1993 oct; 68(10): 784-791.

7. Schmidt HG, Boshuizen HPA. On acquiring expertise in medicine [Internet]. Educational Psychology Review. 1993; 5: 205-221. Available from: http://dx.doi. org/10.1007/bf01323044

8. Nizamuddin SL, Nizamuddin J, Mueller A, Ramakrishna $\mathrm{H}$, Shahul SS. Developing a Hypothesis and Statistical Planning. J Cardiothorac Vasc Anesth. 2017 oct; 31(5): 1878-1882.

9. García CAC. Medicina basada en la evidencia: Fundamentos y su enseñanza en el contexto clínico. 2015; 211.

10. Elstein AS, Schwartz A. Clinical problem solving and diagnostic decision making: selective review of the cognitive literature. BMJ. 2002 mar 23; 324(7339): 729-732.

11. Bckenholt $U$, Weber EU. Toward a Theory of Hypothesis Generation in Diagnostic Decision Making (Internet). Investigative Radiology. 1993; 28: 76-80. Available from: http://dx.doi.org/10.1097/00004424-199301000-00020

12. Melo M, Scarpin DJ, Amaro E, Passos RBD, Sato JR, Friston KJ, et al. How doctors generate diagnostic hypotheses: a study of radiological diagnosis with functional magnetic resonance imaging. PLoS One. 2011 dec 14; 6(12): e28752.

13. Carlin JB, Doyle LW. Statistics for clinicians: 4: Basic concepts of statistical reasoning: hypothesis tests and the t-test. J Paediatr Child Health. 2001 feb; 37(1): 7277.

14. Banerjee A, Chitnis UB, Jadhav SL, Bhawalkar JS, Chaudhury S. Hypothesis testing, type I and type II errors. Ind Psychiatry J. 2009 jul; 18(2): 127-131.

15. Buchinsky FJ, Chadha NK. To P or Not to P: Backing Bayesian Statistics. Otolaryngol Head Neck Surg. 2017 dec; 157(6): 915-918. 
16. Biau DJ, Jolles BM, Porcher R. $P$ value and the theory of hypothesis testing: an explanation for new researchers. Clin Orthop Relat Res. 2010 mar; 468(3): 885-892.

17. Taucher E. Bioestadistica. Editorial Universitaria; 1997; 310.

18. Abramson EL, Paul CR, Petershack J, Serwint J, Fischel JE, Rocha M, et al. Conducting Quantitative Medical Education Research: From Design to Dissemination.
Acad Pediatr. 2018 mar; 18(2): 129-139.

19. Harasym PH, Tsai T-C, Hemmati P. Current trends in developing medical students' critical thinking abilities. Kaohsiung J Med Sci. 2008 Jul; 24(7): 341-355.

20. Akyüz Hi, Samsa S. The effects of blended learning environment on the critical thinking skills of students (Internet). Procedia - Social and Behavioral Sciences. 2009; 1: 1744-1748. Available from: http://dx.doi. org/10.1016/j.sbspro.2009.01.308 